\title{
Duración y valoración en la expresión del pasado: implicaciones para la clase de español como segunda lengua ${ }^{1}$ \\ (Duration and Judgments in Discussing the Past: Implications for the Spanish as a Second Language Classroom)
}

Asunción Martínez-Arbelaiz²

University Studies Abroad Consortium, San Sebastián, España

Isabel Pereira-Rodríguez ${ }^{3}$

New York University, Madrid, España

\section{resumen}

En este estudio se analiza el uso del imperfecto en las narraciones de un grupo de estudiantes universitarios, antes y después de un curso de español en situación de inmersión. Aunque la cantidad de errores de imperfecto disminuye en las categorías observadas, se muestra que, a pesar de la instrucción recibida y de la exposición natural al input, persisten ciertos tipos de error en las narraciones, en particular los errores de uso de imperfecto relacionados con los conceptos de duración y valoración.

\section{abstract}

In this study the use of the imperfect tense is analyzed in the compositions of a group of college students, before and after a Spanish course received

1 Recibido: 05 de diciembre de 2014; aceptado: 06 de enero de 2015.

2 Universidad del País Vasco. Correo electrónico: asuncion@usac.unr.edu

3 Correo electrónico: ip15@nyu.edu 
in an immersion setting. Although the number of errors of the imperfect diminishes in the categories observed, it was shown that, despite the instruction received and the natural exposure to input, several types of errors are still present in the narratives. Errors in the use of imperfect related to the concepts of duration and judgment are particularly persistent.

Palabras clave: español como segunda lengua, análisis de errores, aspecto verbal

Keywords: Spanish as a second language, error analysis, verb aspect

\section{Introducción}

Uno de los asuntos más difíciles que afrontan los profesores de español como segunda lengua o lengua extranjera es explicar la diferencia entre el uso del (pretérito) imperfecto y del (pretérito) perfecto simple, o el denominado pretérito indefinido o simplemente indefinido. Por lo general, los libros de texto utilizados por los alumnos estadounidenses en su primer año de contacto con el español comienzan introduciendo el uso del imperfecto para describir costumbres y hábitos en el pasado, para luego detallar el uso de este tiempo para describir personas y cosas dentro de un contexto pasado. Asimismo, los manuales indican que se usa el imperfecto para acciones repetidas, inacabadas o que duran. De esta forma, el estudiante va forma una idea del uso del imperfecto que les lleva a la producción de oraciones con errores como el que sigue:

\section{(1) *Hacíamos el viaje por avión por más de veinte horas.}

En un estudio previo ${ }^{4}$, se constató un aumento estadísticamente significativo del uso del imperfecto en las narraciones escritas por estudiantes de quinto semestre de español, después de una temporada de estancia en España. Además, se comprobó una disminución de

4 Asunción Martínez Arbelaiz e Isabel Pereira-Rodríguez, «La adquisición del pretérito imperfecto en situación de inmersión», Spanish in Context V,2 (2008): 161-181. 
los usos incorrectos. En el presente estudio analizamos estos errores de uso del imperfecto en las narraciones de nuestros alumnos con objeto de detectar patrones de error y averiguar si hay una explicación subyacente que dé cuenta de estos, con el fin de ser conscientes como docentes de los errores conceptuales de nuestros alumnos. Así, podremos contrarrestar mejor las tendencias originadas, bien como estadios naturales de la adquisición, o por hipergeneralizaciones de la instrucción recibida.

\section{La enseñanza del imperfecto en los libros de texto y gramáticas del español}

La explicación más extendida del uso del imperfecto suele destacar su valor aspectual de acción repetida o durativa respecto a la acción puntual expresada por el perfecto simple, especialmente en niveles iniciales del español. En general, los textos utilizados en el primer año de contacto con el español comienzan ilustrando el imperfecto como equivalente a $I$ used to; es decir, remitiéndose a acciones habituales en un periodo de la vida de las personas. Así, las primeras actividades relacionadas con el imperfecto piden al alumno que piense en las cosas que hacía de niño, en la rutina del semestre anterior, etc., haciendo alusión a la equivalencia del imperfecto con en inglés used o would. Reproducimos a continuación las primeras explicaciones gramaticales que se ofrecen en algunos libros de primer año de amplia difusión en universidades estadounidenses.

- ¿Sabías que..?5: «Spanish also uses the imperfect to refer to actions and events that occurred repeatedly in the past, without reference to exactly how often. This corresponds roughly to English used to or would as in They used (would) make fun of

5 Bill VanPatten, James F. Lee y Terry L. Ballman, ¿Sabías que...?: Beginning Spanish (Boston: McGraw-Hill, 2008) 165-166. 
me as a child. Algunas preguntas: ¿Qué cosas hacías tú de niño (as a child) que no haces de adulto?

- ¿Qué tal?6: «In contrast to the preterit, the imperfect tense is used when you view actions or states of being habitual or as in progress».

- Exploraciones ${ }^{7}$ : «One of the uses of the imperfect is to describe past habits or routines. In English, we frequently use the expression used to. It is often used with expressions such us siempre, todos los días, todos los años, con frecuencia, a menudo (often), normalmente, generalmente, a veces, etc.»

- Dos mundos ${ }^{8}$ : «Saying what you used to do: the imperfect tense. The Spanish imperfect tense is used to describe actions that occurred repeatedly or habitually in the past. To express the same idea, English often uses the phrases used to or would, or just the simple past».

- $\quad$ Encuentros ${ }^{9}:$ «Talking about past routine. Imperfect of Regular -ar Verbs. Spanish has two simple past tenses: the preterit, which you have already learned, and the imperfect. The imperfect is used to talk about repetitive past action and to describe how life used to be. The imperfect has two forms. (...) Práctica y conversación. A. La rutina de Carlos. Un(-a) compañero(-a) de clase y Ud. comparan la rutina de Carlos de este semestre con su rutina del semestre pasado».

Sin embargo, si bien para hablar de acciones habituales lo más utilizado en cuanto a frecuencia es el imperfecto, el perfecto simple

6 Thalia Dorwick, ¿Qué tal?: An introductory course (Boston: McGraw-Hill, 7a. ed, 2007)235.

7 Mary Ann Blitt y Margarita Casas, Exploraciones (Boston: Heinle Cengage Learning, 2012) 274.

8 Tracy D. Terrell, Magdalena Andrade, Jeanne Egasse y Elías Miguel Muñoz, Dos mundos (Boston: McGraw-Hill, 5a. ed., 2002; o 7a. ed., 2010) 309.

9 Emily Spinelli y Marta Rosso-O’Laughlin, Encuentros (Boston: Harcourt College Publishers, 2001) 298-299. 
también alude a actividades habituales o prolongadas en el pasado. En la gramática de Butts y Benjamin ${ }^{10}$ se explica esta posibilidad:

El pretérito ve el evento como ocurriendo de forma ininterrumpida durante un periodo finito mientras que el imperfecto lo describe como ocurriendo en el tiempo o como parte del trasfondo contra el cual se narran los eventos. En la oración Mi padre fumaba/fumó mucho cuando era joven los dos tiempos son posibles, independientemente de que él continuara fumando después o de si todavía vive. El imperfecto ve el hábito en progreso en ese tiempo, el pretérito lo recuerda como un evento visto como una totalidad, es decir, como algo que continúa a través del periodo de tiempo que el hablante tenga en mente.

Suele explicarse el imperfecto aduciendo que se emplea para acciones que duran, que se repiten, inacabadas o que se dan en su desarrollo (no puntuales), como intervalos de tiempo frente al momento puntual del evento expresado con el perfecto simple. Sin embargo, la idea de duración, repetición, no puntualidad no se debe tan sólo al empleo del imperfecto, sino a una coincidencia de varios elementos, entre los que desempeñan un papel fundamental el contexto, el conocimiento que tienen el hablante y el oyente del mundo con todas las experiencias que comporta, en algunos casos la presencia de determinadas expresiones temporales, y el semantismo de cada verbo. En el ejemplo (2), la idea de repetición se debe a la combinación de nuestro conocimiento de lo que es ir en metro, con expresiones como en aquella época o siempre:

(2) En aquella época iba siempre en metro, porque no tenía coche.

La idea de duración, asociada a no tenía coche, no es sino una consecuencia de la combinación de en aquella época con nuestra

10 John Butt y Carmen Benjamin, A New Reference Grammar of Modern Spanish (Malta: McGrawHill, 2000) 206. 
experiencia de tener coche. Se refiere a una situación (idea de duración), según Matte Bon, y no a un acto puntual ${ }^{11}$. No obstante, como señala Brucart ${ }^{12}$, el término «duración» identificado con el imperfecto puede dar lugar a un equívoco si el hablante no considera el hecho sin delimitación temporal:

Si se le quiere otorgar una categorización aspectual, el concepto que debe ser utilizado no es el de duración sino el de no acotación o no terminación, una noción que tiene que ver con la falta de delimitación temporal del evento. De hecho, el propio término de «imperfecto» (que etimológicamente significa «no acabado») alude a este valor ${ }^{13}$.

Según la distinción de Brucart, la duración o la repetición de la acción en los ejemplos en (3), se diferencia por el contraste aspectual entre el evento acotado o no acotado; es decir, por la delimitación temporal del perfecto simple o del imperfecto respectivamente:
a. Fuimos tres veces a Los Ángeles
b. Íbamos tres veces a Los Ángeles cada año
c. Estudié historia en la universidad
d. Entonces estudiaba historia en la universidad

En su gramática comunicativa, Matte Bon plantea otra explicación basada en la opción del hablante de relatar ciertos sucesos como secundarios:

11 Francisco Matte Bon, Gramática comunicativa del español. De la lengua a la idea. Tomo I (Madrid: Edelsa, 1999) 26-27.

12 José María Brucart, «El valor del imperfecto de indicativo en español». Primer Congreso Internacional de la Asociación Coreana de Hispanistas (Corea: Universidad de Chonbuk, 2001). En el presente estudio se analizan los usos más comunes del imperfecto en narraciones; por ello quedan fuera usos pragmáticos modales o metafóricos del imperfecto, como aquellos señalados por Brucart en los siguientes ejemplos: a. Ya nos íbamos; b. Quería pedirle un favor; c. Si me tocara la lotería me compraba un yate; d. Mañana íbamos al cine; e. Perdón, ¿cómo se llamaba usted?

Brucart, 3. 
El enunciador emplea el imperfecto en lugar del pretérito indefinido para referirse a sucesos pasados que no le interesa relatar en sí, sino tan sólo en la medida en que constituyen rasgos o características de una situación que está tratando de reproducir lingüísticamente ${ }^{14}$.

Por tanto, el contraste tiene un trasfondo discursivo en el que el imperfecto representa el valor estático en la narración que suele identificarse con la imagen detenida en un proyector. Por el contrario, los hechos que suceden y se narran se expresan con el perfecto simple. En una distinción entre el valor estático y el valor dinámico de estos tiempos se hace hincapié en la Gramática básica del estudiante de español, en que se explica que el imperfecto y el perfecto simple sirven en español para distinguir cualidades estáticas y cualidades dinámicas de eventos del pasado ${ }^{15}$. Las cualidades estáticas se refieren a la descripción de una imagen de objetos o personas, y se usa por tanto el imperfecto. De ahí que el ejemplo (4.a) sea correcto y el (4.b) de imposible realización. Por el contrario, al referirnos a las cualidades dinámicas de un evento, hablamos de su proceso de principio a fin. Por esta razón, (4.c) es posible y (4.d) no lo es:
a. La mujer que conocí ayer era muy bonita
b. *La mujer que conocí ayer fue muy bonita
c. El concierto de ayer fue muy bonito
d. *El concierto de ayer era muy bonito

Los autores matizan que incluso, «[s]i nos referimos a la duración total de una cualidad (una hora, dos semanas, todo el día, mucho tiempo, etc.) entonces hablamos de un proceso completo yel Indefinido es la única opción ${ }^{16}$. El ejemplo siguiente ilustra esta posibilidad:

14 Matte Bon, 26-27.

15 Rosario Alonso Raya, Alejandro Castañeda Castro, Pablo Martínez Gila, Lourdes Miquel López, Jenaro Ortega Olivares y José Plácido Ruiz Campillo, Gramática básica del estudiante de español (Barcelona: Difusión, 2005) 130.

16 Alonso Raya y otros, 131. 
(5) Tuve el pelo largo dos años, pero luego me cansé

Volviendo a Brucart, el autor destaca que el valor básico del imperfecto es el de coincidir con una referencia pasada. Desde esta perspectiva, usamos el imperfecto para mostrar el contexto en el que irrumpe otro evento, como en (6.a), en contraste con (6.b) donde las acciones se suceden temporalmente:

(6) a. Cuando comíamos, llamó Jorge

b. Cuando terminamos de comer, llamó Jorge

En resumen, la disparidad de explicaciones y la amplia variedad de conceptos que las gramáticas manejan (cf., habitualidad, repetición, acotación, duración, etc.) hacen que no sorprenda que los alumnos de habla inglesa tengan dificultades a la hora de elegir entre el perfecto simple y el imperfecto. Para autores como Ruiz Campillo ${ }^{17}$ o Castañe$\mathrm{da}^{18}$, frente a una larga lista de reglas para explicar el uso y contraste entre estos dos tiempos verbales, conviene acudir a la oposición de «terminado/no terminado», de fácil representación mental y también que permite entender usos discursivos concretos ${ }^{19}$. Ruiz Campillo subraya que el imperfecto sitúa la narración «dentro de la escena, sin interés en la terminación y aporta un valor fotográfico como parte de una secuencia, mientras que el perfecto simple constata el hecho terminado, desde fuera y representa una secuencia completa» ${ }^{20}$.

$\mathrm{Al}$ examinar la producción del imperfecto en la interlengua de alumnos de español parece que la elección del imperfecto relacionado con su valor aspectual durativo, su valor descriptivo y su valor

17 José Plácido Ruiz Campillo, «Instrucción indefinida, aprendizaje imperfecto. Para una gestión operativa del contraste imperfecto/indefinido en clase», Mosaico XV (2005): 8-17.

18 Alejandro Castañeda Castro, «Aspecto, perspectiva y tiempo de procesamiento en la oposición imperfecto/indefinido en español. Ventajas explicativas y aplicaciones pedagógicas», RaeL Revista Electrónica de Lingüística Aplicada 5 (2006): 107-140.

19 Castañeda, 133.

20 Ruiz Campillo, 15. 
de copretérito presenta poca dificultad. Sin embargo, estos alumnos extienden la elección del imperfecto a otros casos que asocian con el posible carácter durativo o estático del evento, como explicamos en la siguiente sección. Por tanto, en esta circunstancia el imperfecto irrumpe en el terreno del perfecto simple, produciéndose el error.

\section{El uso del imperfecto en la interlengua de los alumnos}

Fernández ${ }^{21}$ lleva a cabo un exhaustivo estudio de los errores gramaticales de cuatro grupos de aprendices de español cuya L1 eran el japonés, el alemán, el árabe y el francés repartidos en tres niveles de aprendizaje de $2^{\circ}, 3^{\circ}$ y $4^{\circ}$ curso de La Escuela Oficial de Idiomas de Madrid. Sus datos provienen de redacciones en las que el alumno explica un viaje. En su análisis sobre uso del imperfecto concluye que los errores se deben en su gran mayoría al uso del imperfecto en lugar del perfecto simple. Lo explica como resultado de hipercorrección por parte del alumno, que le lleva a elegir la forma de imperfecto como más característica o peculiar de la lengua meta. Además, señala que el error también proceda del hecho de que el paradigma verbal del imperfecto presenta para elalumno menor dificultad que eldel perfecto simple, haciendo, por tanto, más asequible su uso ${ }^{22}$. Concluye que los errores constatados en su trabajo muestran que la interiorización del alumno de la regla del uso del imperfecto falla porque la captación del valor relativo del imperfecto como información contextualizadora se confunde con la idea de duración y repetición.

Tales datos muestran que los alumnos tienden a usar el imperfecto erróneamente con verbos cuyo lexema es marcadamente permanente, como ser, tener, estar, pasar, hablar, etc., o los verbos de sentimiento o estado de ánimo como gustar, sentir, o encantar. Esta investigación

21 Sonsoles Fernández, Interlengua y análisis de errores en el aprendizaje de español como lengua extranjera (Madrid: Edelsa, 1997).

22 Fernández, 137. 
no contó, sin embargo, con participantes de lengua materna inglesa, aunque todo hace suponer que no se comporten de diferente manera.

Finalmente, Fernández señala que el concepto de «duración» asociado categóricamente al imperfecto es un error inducido probablemente por una simplificación metodológica de los manuales de texto y la didáctica de las propias aulas ${ }^{23}$. Sus informantes confirmaron que los conceptos de «duración»y «acción repetida» se asociaban con el uso del imperfecto, como se muestra en la siguiente cita:

Se reconoce un aspecto imperfecto que se asocia, preferentemente, a un valor de «duración» fuertemente interiorizado, y al de acción repetida en el pasado. Estos dos valores, sobre todo el de duración priman por encima de cualquier otra consideración: acción acabada, inacabada o en desarrollo. De acuerdo con ello, las expresiones temporales (durante las vacaciones, el día, la noche, desde...hasta..., etc.) y las partículas cuantitativas - determinantes o adverbios (mucho, bastante, más, todo) - arrastran el uso del Pr. imperfecto, pues implican la idea de que la acción $«$ dura $»^{24}$.

Como lo explica Fernández, la sintaxis de los tiempos verbales suele presentarse precedida de un capítulo general en el que se constata la importancia de la división entre tiempos absolutos (información central) y relativos (en su valor de contextualizadores o copretéritos). Fernández enfatiza que muchas de las gramáticas pedagógicas de español para extranjeros omiten estas consideraciones previas e insisten en el valor de duración y de acción repetida del imperfecto; inclu so es posible que el propio profesor acentúe esa tendencia.

En un estudio de metodología diferente, Liskin-Gasparro ${ }^{25}$ se indaga en las impresiones que los aprendices de nivel avanzado de español tienen sobre por qué eligen el imperfecto o el perfecto

23 Fernández, 128.

24 Fernández, 137.

25 Judith Liskin-Gasparro, «The Use of Tense-Aspect Morphology in Spanish Oral Narratives:

Exploring the Perceptions of Advanced Learners», Hispania 83, 4 (2000): 830-844. 
simple. Por medio de entrevistas, identifica la razón de la elección de los tiempos del pasado en el propio discurso oral grabado de los informantes y logra hacer explícitas alguna de las razones que guían su uso. En concreto, uno de los participantes de este estudio menciona el test de la interrupción para el imperfecto, en la que se pregunta si la acción fue interrumpida por otra acción. La acción que interrumpe sería la que se conjugaría en perfecto simple. El mismo informante aporta una segunda explicación que gobierna la elección: el test de la rapidez de la acción. Por tanto, si la acción es rápida y no dura mucho, entonces se debe conjugar en el perfecto simple. Este tipo de explicaciones informales en forma de test son las que ayudan y dirigen a nuestros alumnos en sus producciones orales y seguramente también en las escritas.

Una de las principales fuentes de inspiración de los alumnos al formular este tipo dehipótesis ygeneralizaciones son sus libros de texto. Frantzen ${ }^{26}$ estudia la información que se le proporciona al alumno en más de treinta libros de español publicados en los EE.UU. y observa que los manuales tienden a dar generalizaciones simplificadas, o lo que en inglés se llama rules of thumb, las cuales podrían matizarse o suprimirse en algunos casos por llevar al alumno a formarse falsas explicaciones del fenómeno en cuestión, como la autora muestra con profusión de ejemplos. Entre estas generalizaciones están las siguientes: 1) El imperfecto se usa para expresar acciones repetidas o habituales pasadas; y 2) Would + infinitivo señala el uso del imperfecto. Frantzen propone dividir la primera regla en dos, puesto que el concepto de habitualidad no es sinónimo del de repetición. Una acción puede ser habitual pero no repetida y viceversa, por lo que señala que se debería explicar de forma separada el uso del imperfecto para acciones habituales del uso del imperfecto para acciones repetidas en el pasado. En cuanto a la posibilidad de traducir would + infinitivo por el imperfecto, 
esta afirmación sólo es cierta cuando se trata de acciones habituales, por lo esta generalización se podría subsumir en la anterior.

Frantzenconcluye queestas generalizaciones deberían matizarse en algunos casos y sustituirse en otros, por explicaciones que aborden los principios subyacentes que gobiernan la elección del perfecto simple y el imperfecto. De no completar estas reglas con práctica y discusiones orales, éstas podrían confundir y frustrar a los alumnos.

En suma hay estudios previos que insisten en que los alumnos de español como segunda lengua continúan usando el imperfecto en situaciones en las que deberían haber utilizado el perfecto simple y que tanto las reglas que estos formulan como los libros de texto pueden propiciar la aparición de dicho error. En el presente estudio se ahonda en el conocimiento de los errores en el uso del imperfecto en la producción escrita de alumnos estadounidenses de español de tercer año, ya que en el estudio de Fernánde $z^{27}$ no se incluyeron hablantes de lengua inglesa. Nos planteamos la siguiente pregunta de investigación: ¿Qué tipo de de errores cometen los aprendices estadounidenses de tercer año de español en el uso del imperfecto antes y después de un semestre de instrucción en España?

\section{Diseño del estudio}

Los participantes redactaron una narración personal sobre un viaje al principio de su quinto semestre de español y otra al finalizar su estancia en España, al término de un programa de quince semanas. En las instrucciones se les pedía que contaran dicho viaje en 250 palabras aproximadamente en media hora de clase. El tema elegido procuraba estimular el uso de los tiempos del pasado por parte de los alumnos. Elegimos la producción escrita y no la oral porque pensamos que el enfoque momentáneo en la forma que puedan realizar los alumnos será más evidente en el output escrito que en el oral, dado que pueden

27 Fernández. 
detenerse y reflexionar sobre la elección entre el perfecto simple y el imperfecto. La capacidad de narrar y describir en el pasado es un rasgo que caracteriza a los aprendices de nivel avanzado según las ACTFL Proficiency Guidelines ${ }^{28}$ y el nivel B1 o umbral en el Marco Común Europeo de Referencia ${ }^{29}$.

\section{Participantes}

Un total de treinta y siete alumnos completaron las dos composiciones requeridas. Se eliminaron los casos de alumnos de procedencia hispana, aquellos que habían participado en programas de inmersión con anterioridad y los que no completaron las dos composiciones. Los alumnos eran estudiantes de diversas universidades de EE.UU., que asistían al programa de inmersión de la New York University en Madrid o a los programas de la University Studies Abroad Consortium (de la University of Nevada, Reno) en San Sebastián y Madrid. Recibieron clases de español centradas en la mejora de aspectos gramaticales y discursivos de lunes a jueves.

El tiempo real que la asignatura dedicó a la enseñanza del pasado no superaba tres horas de duración, aunque la precisión gramatical en los usos de los tiempos pasados fuera uno de los objetivos a lo largo del curso en diferentes actividades tanto orales como escritas. Además de las horas de contacto con la lengua meta en el aula, los alumnos tuvieron la oportunidad de interactuar con hablantes nativos en sus actividades diarias. La mayoría de los alumnos de inmersión habían establecido relaciones amistosas por medio de un servicio de intercambio de idiomas de la universidad, participaban en deportes, vivían con familias o con otros alumnos de su edad. Sin embargo, no se tuvo en cuenta la variedad individual en cuanto al uso real que hicieron de estasoportunidades.

28 Elvira Breiner-Sanders, Karen Swender y Robert M. Terry, Preliminary Proficiency Guidelines. Writing, 2001; disponible en: 〈http://www.actfl.org/files/public/writingguidelines.pdf $>$.

29 VV.AA. Marco Común Europeo de Referencia para las Lenguas: Aprendizaje, Enseñanza y Evaluación, 2002; disponible en: 〈http://cvc.cervantes.es/ensenanza/biblioteca_ele/marco〉. 


\section{Recogida y codificación de datos}

Las redacciones se llevaron a cabo en el aula y al profesor de cada curso se le proporcionó una serie de instrucciones para evitar que algunos alumnos consultaran diccionarios, hicieran preguntas al profesor o excedieran los treinta minutos de tiempo asignado. Al codificar los datos según el tipo de error, se llegó a una clasificación o tipología de errores en la que se tuvo en cuenta tanto los conceptos recogidos en las gramáticas pedagógicas y libros de texto, como las observaciones de Fernández $z^{30}$ y de Liskin-Gasparro ${ }^{31}$. Además, se añadieron nuevos patrones de error que emergieron en nuestros datos. Finalmente, se formuló la siguiente tipología que, sin ser exhaustiva, da cuenta precisa de los patrones de error hallados:

- Valoración.

- Duración con verbos de aspecto léxico no puntual.

- Formas verbales acompañadas de una expresión adverbial durativa.

- Formas verbales acompañadas de un complemento con significado de «mucho».

- Expresiones con cuando/mientras.

- Imperfectos que debían haber sido subjuntivos u otros casos.

\section{Resultados}

Con el estudio se comprobó un notable aumento en la producción de formas del imperfecto, elevándose de 319 instancias producidas al comienzo del curso a 574 al final. Es decir, los alumnos aumentaron su producción de imperfectos en un 44,42\%. En cuanto a los errores cometidos al principio y al final de semestre, observamos que el porcentaje de usos incorrectos disminuyó al término de su estancia en España. Así, al comenzar el semestre, de las 319 instancias constatadas, 47 fueron incorrectas, es decir, el porcentaje de error fue de $14,7 \%$. Sin

30 Fernández.

31 Liskin-Gasparro. 
embargo, concluido el programa, fueron 38 las instancias incorrectas de un total de 574; disminuyendo, por lo tanto a un 6,62\% el índice de error. Resumimos estos resultados en el cuadro 1 y presentamos a continuación los resultados obtenidos de cada tipo de error establecido en nuestra clasificación.

\section{Cuadro 1. Uso del imperfecto y errores antes y después de su estancia en España}

\begin{tabular}{|c|c|c|c|c|c|c|c|}
\hline \multicolumn{2}{|c|}{$\begin{array}{c}\text { Imperfectos } \\
\text { Antes }\end{array}$} & \multicolumn{3}{c|}{$\begin{array}{c}\text { Imperfectos } \\
\text { Después }\end{array}$} & $\begin{array}{c}\text { Incremento } \\
\text { en uso }\end{array}$ & $\begin{array}{c}\text { Disminución } \\
\text { del índice de } \\
\text { error }\end{array}$ \\
\hline Total & Errores & \% & Total & Errores & \% & \% & \% \\
\hline 219 & 47 & 14,7 & 574 & 38 & 6,62 & 44,42 & 6,62 \\
\hline
\end{tabular}

\section{Valoración}

El concepto de valoración arroja como resultado errores en el uso del imperfecto en lugar del perfecto simple. En nuestros datos, estos errores se producen en la valoración o evaluación del evento de viaje en sí mismo, como se observa en (7):

(7) a. *Para mí este viajar era lo mejor porque aprendí mucho de la cultura, de la vida, y también divertirme

b. *Era un viaje memorable!

Antes de su estancia en España, el número de errores de este tipo fue 17 de los 47 errores cometidos; un porcentaje de 36,1\% del total de errores. Tras su estancia en España fueron 8 de 38 los errores constatados, y por lo tanto este tipo de error representó el $21 \%$ del total. Respecto al total de instancias del imperfecto, el porcentaje de errores de este tipo fue 5,5\%, mientras que tras su estancia disminuyó al 1,3\%. 
Como señalan Alonso Raya y otros ${ }^{32}$, la valoración es la recuperación del pasado de una imagen o secuencia de imágenes vistas de principio a fin y que, por lo tanto, deberían estar en perfecto simple. La valoración hace referencia a la cualidad dinámica del evento, evaluándolo desde el principio al fin (ej. El concierto de ayer fue mиy bonito), mientras que el imperfecto se usaría para la descripción de cualidades estáticas de una imagen, persona u objeto (La mujer que conocí ayer era muy bonita). Por tanto, si se toma como ejemplo una fiesta «el imperfecto nos dice que el hablante se coloca dentro de la fiesta para describírnosla; el indefinido, que el hablante se coloca fuera $»^{33}$. En los ejemplos se constata que el alumno se fija quizás en el aspecto durativo, como por ejemplo, de una fiesta o un viaje, o descriptivo sin percibir que la valoración en español se expresa en términos absolutos, como ya señalaba Fernández ${ }^{34}$.

Uno de los dos grupos utilizó un libro de texto en el que se explica el uso del perfecto simple como valoración. Sorprendentemente este grupo no es el causante de la disminución en el índice de error respecto a la valoración, ya que dicho grupo, formado por 12 alumnos, registró cuatro errores al principio y otros cuatro al final del tratamiento. No es posible confirmar que haya habido una influencia del libro de texto en este aspecto en particular.

\section{Duración con verbos de aspecto léxico no puntual}

Por verbos de aspecto léxico no puntual entendemos aquellos que describen actividades con duración y se opondrían a los verbos delimitados y de escasa duración, los cuales fueron denominados «logros» (achievements) por Vendler ${ }^{35}$. Según De Miguel $^{36}$, los verbos de aspecto léxico puntual «son los que describen un evento que tiene lugar en un

32 Rosario Alonso Raya y otros, 130.

33 Ruiz Campillo, 8-17.

34 Fernández.

35 Zeno Vendler, Linguistics in Philosophy (Ithaca, Nueva York: Cornell University Press, 1967).

36 Elena De Miguel, «El aspecto léxico», en Ignacio Bosque y Violeta Demonte, eds., Gramática descriptiva de la lengua española 2 (Madrid: Espasa-Calpe, 1999) 73-99. 
instante temporal único y definido, sin fases: alcanzar la cima de un monte, estallar de ira, explotar una bomba, llegar a la meta, marcar un gol, nacer, reconocer una cara, morir» (3033). Aunque de muy reducido uso, estos verbos ocasionalmente podrían usarse en imperfecto. Sin embargo, es en los verbos de aspecto léxico no puntual, bien sean estados, actividades o realizaciones, indica Vendler, donde el alumno tiende a ver un argumento para el uso del imperfecto debido a su «duración», identificándolos con la representación no terminativa. Así, se producen errores como los ejemplos en (8), los cuales supusieron el 1,8 \% y el $1,7 \%$, antes y después del semestre respectivamente.

(8) a. *Ibamos por caballo por los árboles y miramos a todo b. *Normalmente ella es seria pero esa vez nole gritaba al primo.

Formas verbales acompañadas de una expresión adverbial durativa

En este tipo de oraciones el imperfecto aparece acompañado por una «expresión adverbial durativa», ya que ésta indica la cantidad de tiempo que «dura» el evento descrito. Este tipo de error representó el 2,5\% del total de instancias de imperfecto antes de comenzar el semestre y disminuyó hasta el 1,3\% al término del semestre. Los siguientes ejemplos ilustran este tipo de error:

(9) a. *Un día, jugábamos por tres horas

b. *Mi amiga y yo bailábamos por todo el noche.

Según Castañeda ${ }^{37}$, hay una incompatibilidad del imperfecto con complementos adverbiales temporales que expresan límites temporales del proceso y es precisamente esta acotación la que el estudiante confunde con la duración.

37 Castañeda, 109. 


\section{Formas verbales acompañadas de un complemento con significa-} do de «mucho»

La idea de prolongación o duración en el tiempo no solo se expresa mediante frases adverbiales, sino también por medio del complemento directo o del circunstancial. En estos complementos puede incluirse el significado de «mucho» o se da una lista de objetos. El porcentaje de errores de este tipo fue muy pequeño antes y después del semestre, $0,6 \%$ y $0,3 \%$, respectivamente. Ejemplos de estos errores se muestran en las siguientes oraciones:

(10) a. *La próxima día de nuestro fin de semana en Portugal, nosotros caminamos mucho y veíamos muchas cosas.

b. *Nosotros conducíamos a Los Angeles, las Vegas, Utah, New Mexico, Arizona, Texas, New Orleáns, etc.

\section{Expresiones con cuando/mientras}

Los errores de este tipo se atribuyen a un error en la asignación temporal de los verbos para que uno indique la acción interrumpida por otro, apareciendo ambos en imperfecto o en el orden inverso al correcto, como se muestra respectivamente en los ejemplos a continuación.

\section{(11) a. *Cuando llegábamos al aeropuerto en Lima, estaba esperán- donos mi tío. \\ b. Nosotros rompíamosnuestrocochecuando estuvimosenlaplaya.}

Los alumnos comenzaron el semestre cometiendo un 2,5 \% de errores de este tipo, mientras que al cabo del semestre, el porcentaje de error disminuyó hasta representar solo el 1,3\% de las instancias totales en el uso de los pasados. Como explica Ruiz Campillo ${ }^{38}$, es la duración del acto la que lleva al alumno a este tipo de error en lugar de advertir que se está fuera de un proceso completo, y, por tanto,

$38 \quad$ Ruiz Campillo. 
la forma verbal correcta en los ejemplos en (11) sería «llegamos» y «rompimos» respectivamente.

\section{Imperfectos que debían haber sido subjuntivos u otros casos}

En nuestros datos el porcentaje de errores por la confusión del imperfecto con el imperfecto de subjuntivo, como se ilustra en el ejemplo (12), fue muy bajo antes y después de la estancia de nuestros alumnos en España, 0,6 \% y 0,3 \% respectivamente.

(12) *Me gusto que me llevaba en la moto porque me dio la vista del campo...

Solo hubo una instancia que no correspondía con un error de subjuntivo, por eso la sumamos a este grupo y no hicimos otro apartado para otros casos.

Resumimos en el cuadro 2 los resultados por tipos de error y en su conjunto. También mostramos el número de errores, según hayan ocurrido antes o después del semestre en inmersión.

\section{Cuadro 2. Número de instancias y porcentajes para cada tipo de error}

\begin{tabular}{|l|c|c|c|c|}
\hline \multicolumn{1}{|c|}{ Tipo de error } & Antes & \% & Después & \% \\
\hline Valoración & 17 & 5,5 & 8 & 1,3 \\
\hline Léxico no puntual & 6 & 1,8 & 10 & 1,7 \\
\hline Adv. durativo & 8 & 2,5 & 8 & 1,3 \\
\hline Mucho & 6 & 1,8 & 2 & 0,3 \\
\hline Cuando/mientras & 8 & 2,5 & 8 & 1,3 \\
\hline Otros & 2 & 0,6 & 2 & 0,3 \\
\hline Total & $\mathbf{4 7}$ & $\mathbf{1 4 , 7}$ & $\mathbf{3 8}$ & $\mathbf{6 , 2}$ \\
\hline
\end{tabular}

Eluso del imperfecto erróneo disminuye tanto en número totalde instancias, puesto que baja de 47 a 38, como en porcentaje, el cual baja de $14,7 \%$ a $6,2 \%$. Los errores que se reducen después de la estancia 
en España son la valoración y las formas verbales acompañadas de un complemento con significado de «mucho». Aumenta levemente el número de errores del tipo duración con verbos de aspecto léxico no puntual, mientras que el resto de las categorías presentadas se mantienen igual. Si bien hay que señalar que los porcentajes antes y después de la estancia en España muestran variaciones escasamente perceptibles, lo importante es observar que el patrón de error en el uso del imperfecto se mantiene en las mismas categorías observadas al principio del estudio.

\section{Discusión e implicaciones pedagógicas}

En este estudio se comprueba que, transcurrido un semestre en situación de inmersión, el porcentaje de error se reduce considerablemente (de $14,7 \%$ a $6,2 \%$ ), pero también se mantiene la misma tendencia al error debido a la percepción que el alumno tiene sobre la duración y la valoración del evento que describe. La idea de duración, relacionada con el significado del lexema verbal, con los adverbios durativos y con objetos directos que apuntan a la idea de que mucha cantidad desencadena el uso del imperfecto. Además, la distribución aspectual en oraciones que incluyen una subordinada temporal (ej. mientras..., cuando...) presenta dificultades de expresión para los aprendices. Tales resultados, similares a los de Fernández ${ }^{39}$, indican que los errores son reflejo de evoluciones universales en la adquisición de los valores aspectuales de las formas verbales del pasado. Además, a pesar de la disminución de errores tan notable después de un semestre en España, tampoco parece claro que hayan alcanzado en su uso del imperfecto el nivel de sofisticación que sugiere el plan curricular del Instituto Cervantes para el nivel B2.

Como lo indica Fernánde $z^{40}$, una consideración pedagógica a la que nos llevan estos errores es el no insistir en la clase en la idea «de 39 Fernández.

40 Fernández, 140. 
acción que dura» para explicar el uso del imperfecto, ya que esto hace que el alumno hipergeneralice esta regla a acciones acotadas. Se debe, según la autora, insistir en el valor absoluto de la acción expresada en indefinido, aunque este momento se refiera a «toda la tarde», o se haya realizado «muchas veces».

Se debe insistir en relacionar la aportación del lexema verbal de aspecto permanente con los valores de acción pasada y temporalmente acotada del perfecto simple. Ejemplos como el que reproducimos a continuación pueden analizarse en clase, de forma que el alumno tome conciencia de que una expresión como «estar lleno», a pesar de que no es una acción y por lo tanto parece tener un aspecto más permanente, puede aparecer en perfecto simple.

Por la puerta del «Río Bar» pasaba mucha gente. Algunos entraban. Pronto, la terraza estuvo llena de hombres y mujeres que hablaban en voz alta y reían ${ }^{41}$.

De este modo el alumno comprueba que el lexema verbal de aspecto no puntualopermanente notienenecesariamente queasociarse con el aspecto verbal durativo que el imperfecto pudiera representar.

$\mathrm{Al}$ igual que con las matizaciones del concepto de duración, el profesor debería insistir en el uso del perfecto simple asociado a la valoración del evento en cuestión; y recalcar que al salir de un cine, de una conferencia, o de cualquier acto, se usa el perfecto simple para valorar esas actividades: Me gustó muchola película, La conferenciafue muy interesante, la fiesta salió muy bien. De ahí que una intervención pedagógica que distinga entre la evaluación/valoración de un evento, situación o actividad y la descripción de situaciones pasadas puede ser eficaz para evitar la tendencia de los alumnos a usar el imperfecto al terminar la narración de su viaje y realizar una valoración de este, como en los ejemplos que vimos.

41 Mario Vargas Llosa, Los jefes (Madrid: Aguilar, 1974) 82. 
Estarea delos profesores contrarrestargeneralizaciones erróneas que inducen al error en el uso del imperfecto en el lugar del perfecto simple, previniendo así el riesgo de su fosilización. Sabemos que la explicación metalingüística por parte del profesor o dellibro de texto no es suficiente para que se produzca la adquisición(cfr.Felix;Pienemann; y $\mathrm{Pica}^{42}$; entre otros). Es necesario que se muestren al alumno estos usos del perfecto simple en un input realzado para que tenga lugar el intake, de acuerdo con Sharwood $S_{m i t h}{ }^{43}$, y romper con la tendencia del alumno a usar el imperfecto. Incluso podría ser insuficiente el realzamiento del input para su internalización y automatización, y por tanto, como Swain ha señalado reiteradamente en el campo de la adquisición ${ }^{44}$, es necesario facilitar oportunidades de producción, tanto controladas (semicomunicativas) como más comunicativas para la consolidación de esta dicotomía aspectual.

Como señala Zanón ${ }^{45}$, el objetivo de las actividades de concienciación gramatical es que el alumno reflexione sobre ciertos aspectos gramaticales, y propiciar el análisis y la comprensión de sus funciones para contribuir así a la representación mental correcta del elemento que se analiza. En este tipo de tareas la comprensión y producción precisas deben ser esenciales para su resolución, deben incorporar un uso comunicativo de la lengua y deben ir acompañadas de un feedback sobre el uso de la estructura dada a lo largo de la actividad. Teniendo en cuenta la dificultad asociada a la idea duración y también

42 Sascha W.Felix, «More Evidence on Competing Cognitive Systems», Second Language Research I, 1 (1995): 47-72; Manfred Pienemann, «Is Language Teachable? Psycholinguistic Experiments and Hypotheses», Applied Linguistics X, 1 (1989): 52-79; Teresa Pica, «The Selective Impact of Classroom Instruction on Second-Language Acquisition», Applied Linguistics VI, 3 (1985): 214-222.

43 Michael Sharwood Smith, «Input Enhancement in Instructed SLA», Studies in Second Language Acquisition XV,2 (1993): 165-179.

44 Merrill Swain, «Communicative Competence: Some Roles of Comprehensible Input and Comprehensible Output in Its Development», en Susan M. Gass y Carolyn G. Madden, eds., Input in Second Language Acquisition (Rowley, MA: Newbury House, 1995) 235-253; Merrill Swain, «The Output hypothesis. Theory and Research», en Eli Hinke, ed., Handbook of Research in Second Language Teaching and Learning (Oxford: Oxford University Press, 1995) 471-484.

45 Javier Zanón, La enseñanza de E/LE mediante tareas (Madrid: Edinumen, 1999) 83-84. 
a la de valoración, los profesores de español debemos diseñar tareas que requieran manipulación y reflexión gramatical de un input rico en estos usos más marcados y complejos de la expresión del pasado.

\section{Conclusión}

Se han analizado algunos de los errores frecuentes en el uso del imperfecto, por parte de quienes aprenden el español como segunda lengua, antes y después de una estancia de quince semanas en España. Nuestros participantes no solo hicieron un uso más extenso de este tiempo verbal, sino que también pudimos verificar que el índice de error disminuyó al cabo de dicha estancia. En cuanto a la naturaleza de los errores cometidos antes del periodo experimental y después de ese periodo, se halló que estos errores respondían a la misma tipología. Esto indica un área de posible fosilización en la interlengua del alumno parcialmente motivada por una percepción errónea del aspecto durativo en la elección de la forma verbal adecuada. Para contrarrestar esa posible fosilización, el profesor debería diseñar una serie de actividades que respondan a cada uno de los errores identificados en los escritos de los alumnos.

En suma, como profesores tales resultados nos hacen pensar en una aproximación metodológica que dé cuenta de esta dificultad que el alumno parece tener para asociar la duración y la valoración con el perfecto simple. Se trata de ayudar al alumno a percibir en el input características gramaticales de índole marcada o compleja y, por tanto, de difícil acceso simplemente con una exposición natural a la lengua meta. Futuros estudios podrían examinar si este tipo de enfoque metodológico centrado en usos específicos de un ítem gramatical determinado tendría una repercusión en la adquisición de los mismos y, así, evitar su fosilización en etapas avanzadas de la adquisición del español. 